Cahier de linguistique

\title{
La traductologie, la traduction naturelle, la traduction automatique et la sémantique
}

\section{Brian Harris}

Numéro 2, 1973

Problèmes de sémantique

URI : https://id.erudit.org/iderudit/800013ar

DOI : https://doi.org/10.7202/800013ar

Aller au sommaire du numéro

Éditeur(s)

Les Presses de l'Université du Québec

ISSN

0315-4025 (imprimé)

1920-1346 (numérique)

Découvrir la revue

Citer cet article

Harris, B. (1973). La traductologie, la traduction naturelle, la traduction automatique et la sémantique. Cahier de linguistique, (2), 133-146.

https://doi.org/10.7202/800013ar d'utilisation que vous pouvez consulter en ligne.

https://apropos.erudit.org/fr/usagers/politique-dutilisation/ 
LA TRADUCTOLOGIE, LA TRADUCTION NATURELLE, LA TRADUCTION AUTOMATIQUE ET LA SÉMANTIQUE ${ }^{1}$

La distinction entre langage et métalangage est bien connue ${ }^{2}$; les notions que sous-tend méta sont importantes pour l'épistémologie de la linguistique. À partir de cette première notion, nous distinguerons maintenant les opérations linguistiques des méta-opérations linguistiques.

Une langue se compose d'éléments de vocabulaire, dans un sens large du terme vocabulaire, et de règles de grammaire. Les éléments peuvent être, soit physiques (par exemple, les sons de la parole), soit abstraits (par exemple, les phonèmes). Ce système reste potentiel, jusqu'au moment où un sujet parlant, émet un énoncé et, de ce fait, met le système en opération. Empruntons un terme à 1'informatique - après tout, quel auteur technique ne parle pas de nos jours du hardware, du software, etc.; à notre tour, nous parlerons de l'implantation du sys tème.

En fin de compte, c'est la parole qui assure la réalité, parce que 1a parole oblige une implantation du système. L'implantation est une opération. Par exemple, l'opération qui consiste à énoncer des sons s'appelle la prononciation. Ainsi, dans la hiérarchie que nous essayons d'établir, l'opération est effectuée par un sujet parlant dans

1. Le point de départ de cette conférence est le livre de A. Ljudskanov, Traduction humaine et traduction automatique, 2 fascicules, Dunod, Paris, 1969.

2. Voir p. 219-240, article de J.-J. Nattiez. 
une situation normale. Il n'est pas nécessaire que le sujet parlant dans une telle situation soit conscient du système qu'il implante. Cette inconscience est une vérité de La Palice en linguistique. Prononciation et phonologie sont, tous les deux, des termes qui appartiennent au métalangage linguistique. Néanmoins, le premier se rattache à une opération linguistique tandis que le second se rattache à une métaopération d'ordre analytique.

La traduction est une opération linguistique de premier niveau, c'est-à-dire une opération pratiquée par le sujet parlant sans même qu'il en soit conscient. La traduction se place ainsi au même niveau que la prononciation. Par contre, si on ne fait pas la traduction, mais par contre on en parle, lorsqu'on analyse en tant que linguiste, ce qu'est la traduction, on atteint alors un niveau égal à celui de la phonologie par rapport à la prononciation. Comment dénommer cette méta-opération?

Dans un autre domaine de la recherche, l'élaboration d'un langage de documentation pour la linguistique ${ }^{3}$, nous avons constaté plusieurs lacunes dans la terminologie de la linguistique dont, justement, le manque d'un terme pour distinguer $I^{\prime}$ analyse linguistique de la traduction. Pour pallier à cette carence, Nida a titré son ouvrage le plus important sur le sujet, The Sciences of Translation 4 ; et Catford a intitule un ouvrage aussi important, The Linguistic Theory of Transla$t_{i o n}{ }^{5}$. De telles périphrases ne font que souligner le besoin d'un terme plus concis. Imaginez-vous, si chaque fois qu'on voulait parler de phonologie, il fallait dire "la science linguistique de la prononciation" :

Nous proposons donc un néologisme pour combler la lacune. Nous conserverons traduction pour l'opération que pratique le traducteur,

3. B. Harris, "A justification and a suggestion for a linguistics". documentation language", Cahiers Zinguistiques d'ott cava, n ${ }^{\circ} 1,1972$.

4. E. A. Nida, Toward a Science of TransZation, Bril1, Leiden, 1964.

5. J. Catford, A Linguistic Theory of TransZation, Oxford University Press, London, 1965. 
mais adopterons traductologie pour toute référence à 1 'analyse linguistique du phénomène.

Dans le langage courant, on emploie traduction dans tous les contextes. En effet, toutes les distinctions conceptuelles nécessaires à cette discussion ne sont pas encore établies : la dichotomie traduction/ traductologie laisse toujours ambigu le mot traduction. Si la traduction est, comme le souligne Ljudskanov, une opération, ce terme signifie également le résultat de cette opération.

Parlons donc de la traduction tout court, c'est-à-dire de 1'opération traduisante, du texte traduit, qui en est le produit, et de 1a traductologie, qui constitue 1'analyse de la traduction, de 1'opération traduisante - analyse linguistique et, éventuellement, psycholinguistique. Ensuite, puisque 1'important dans cette terminologie n'est pas la nomenclature mais la compréhension des concepts qui s'y rattachent, nous nous attaquerons à titre de"traductologue"au concept de traduction.

Ljudskanov, vers la fin du premier fascicule de son ouvrage, ajoute la note suivante :

Grâce à une certaine intuition et à une certaine habitude, chaque sujet bilingue traduit, d'une manière ou d'une autre. Par conséquence, la science de la traduction humaine, en principe, n'avait pas à s'occuper de la question : "Comment apprendre à l'homme à traduire6".

En effet, cette constatation mérite une place de premier plan. On peut l'illustrer par des observations empiriques.

Les enfants d'une famille chinoise d'Ottawa, la famille H., outre le chinois qu'ils connaissent comme langue maternelle, apprennent progressivement $I^{\prime}$ anglais parce qu'ils rencontrent des enfants anglophones et fréquentent la maternelle anglaise. L'aîné parle déjà à cinq ans un anglais presque normal pour un enfant de cet âge, tandis que le cadet, âgé de trois ans ne parle encore que le chinois avec quelques mots iso-

6. A. Ijudskanov, 1968, Traduction humaine et traduction automatique, fascicule 1, p. 50 . 
lés d'anglais. Il est donc possible de bavarder avec l'aîné, mais la barrière des langues rend difficile la communication avec le cadet. Sauf que le problème du cadet est tout de suite résolu lorsque son frère est présent. Chaque fois que le cadet baragouine quelque chose en chinois, il suffit de demander à 1 'aîné : "Que désire ton frère ?"; et pour lui répondre ou lui transmettre une information, il suffit de dire : "Dís cect à ton frère". C'est un canal de communication efficace, qui permet de formuler une hypothèse selon laquelle la faculté de traduction, ou en terminologie chomskienne, la compétence (en traduction), existe déjà chez tout enfant normal et bilingue de cinq ans. ¿̀ quel âge exactement, est-ce que cette compétence s'établit ou s'éclôt chez l'enfant ? Sans pouvoir répondre à cette question, nous voudrions en souligner la pertinence parce qu'elle apportera probablement la découverte que $1 \mathrm{a}$ traduction est une des compétences les plus répandues et les plus élémentaires de tout 1 'éventail de notre comportement linguistique.

Une expérience connexe consistait à demander cette fois à 1'aîné, non pas de dire ce que son frère voulait exprimer, mais de le traduire. Alors il restait embarrassê. On peut en conclure qu'il savait traduire, mais ne connaissait pas le métaterme traduire. On ne l'avait pas rendu conscient de 1'opération qu'il faisait.

À notre première question sur 1 'apprentissage des opérations de traduction, s'en ajoute donc une autre, plutôt piagienne : "À quel âge est-ce que le concept de traduction se forme chez 1'enfant bilingue ?"

Faute d'une documentation plus rigoureuse sur ce qui nous semble un phénomène digne d'intérêt dans 1'apprentissage des langues, revenons plutôt au cas des deux enfants chinois. Considérons la façon de traduire chez 1'aîné. Il ne part pas d'un texte écrit, mais fait le tout par la parole. II ne traduit pas au fur et à mesure que son frère parle; au contraire, il attend la fin de chaque mini-discours pour ensuite en rendre tout le contenu à la fois. Or, dans les écoles de traduction on considère que l'interprétation parlée du discours parlé, que $1^{\prime}$ on appelle $1^{\prime}$ interprétation par opposition à la traduction écrite, 
est plus difficile à pratiquer que la traduction par écrit. Plus encore, l'interprétation simultanée, c'est-à-dire la traduction qui se déroule au moment où 1 'orateur parle (un traitement en temps réel, dirait l'informaticien), est considérée moins difficile que l'interprétation consécutive, c'est-à-dire la traduction faite quand 1 'orateur vient de terminer une séquence. Il est évident que la production consécutive exige un plus grand effort de mémoire. Et bien, des trois façons de traduire que l'on enseigne dans les cours professionels, c'est par la plus difficile que l'enfant chinois débute. Et cet enfant n'est pas exceptionne1.

À côté des "exploits" de ce dernier, nous avons mentionné 1'opinion des traducteurs professionnels. Comme Ljudskanov le montre bien dans la section historique de son livre, 1a traductologie dans le passé était fort colorée par 1 'opinion des traducteurs professionne1s. Sans contester pour le moment leurs opinions, nous voulons quand même éviter la confusion entre la compétence et la performance de 1 'enfant bilingue et le résultat d'une formation poussée et consciente. Par conséquent, $i l$ faut parler, dans le cas de l'enfant chinois, de la traduction naturelle par opposition à la traduction professionnelle. On peut comprendre, par analogie, que le statut de la traduction naturelle vis-à-vis de la traduction professionnelle est semblable à celui de la conversation de tous les jours, par rapport à la composition d'un texte littéraire.

Avant d'estimer 1'importance de la traduction naturelle, une autre nuance à propos du terme traduction s'impose. Puisque l'objet de la linguistique est le langage naturel et humain, le traductologue-1inguiste considère l'opération traduisante comme une traduction d'une langue naturelle et humaine donnée dans une autre. Il est vraí que Ljudskanov renonce explicitement à cette contrainte en tant que sémioticien. On ne doit pas supposer que lorsqu'un sémioticien parle de langage, 1 'usage qu'il fait de ce terme colncide avec celui qu'en fait le linguiste. Il faut inclure dans la circonscription linguistique de 
ce terme des considérations sur les dialectes et les niveaux de langage. La traduction naturelle s'avère des plus usuelles si l'on tient compte de la proportion très considérable de personnes qui habitent dans un milleu bilingue, voire multilingue, si l'on tient compte aussi de la traduction faite à 1 'intérieur de la langue, de $I^{\prime}$ enfant aîné qui aide à interpréter le baragouinage de son cadet, des amis qui nous fournissent des interprétations en français académique des disques d'Yvon Deschamps $^{7}$, et ainsi de suite.

Voici un dernier témolgnage concernant les enfants. Des étudiants à $1^{\prime}$ Université de Montréal ${ }^{8}$ ont fait récemment un sondage local sur la motivation, ou la prétendue motivation, des jeunes qui suivent un cours d'anglais dans plusieurs écoles françaises de la métropole. Parmi les enfants de familles immigrantes, plus précisément italiennes, un mobile fréquent reste que 1 'apprentissage de l'anglais sert à aider les parents ne connaissant pas cette langue. D'une façon intuitive, ces enfants s'imaginent dans le rôle de traducteur pour la famille.

II s'ensuit un argument accablant. Nous avons déjà proposé I'analogie entre la traduction naturelle et la traduction professionnelle d'une part, entre la conversation banale et les belles-lettres d'autre part. Sans vouloir exclure des manifestations de la parole du domaine linguistique, si 1 'on posait à la communauté des linguistes contemporains la question : "Lequel vous intéresse principalement, le langage ordinaire ou celuj des belles-lettres ?", la rêponse serait à 1'unanimité "1e langage ordinaire". Alors, si l'on pose la question analogue par rapport à la traduction, la réponse doit être dans le même sens.

Malheureusement il paraît que cet objet principal de la productologie, voire aucune productologie intéressante du point de vue linguistique, n'est reconnu à 1 'heure actuelle ni par le département de linguistique ni par les cours de production dans nos universités cana-

7. Monologueur du Québec qui exploite le patois.

8. Cours L. Ang. 304, 1972. 
diennes. D'une part et de 1'autre, 1'initiative dans ce domaine se limite aux cours destinés à la formation professionnelle du producteur pratiquant. Certes, de tels cours sont nécessaires, mais il laissent la traductologie deux siècles en arrière par rapport aux autres courants de la linguistique contemporaine. Car le résultat de cette orientation, ce dont Ljudskanov se plaint à juste titre, est une traductologie nettement prescriptive. D'ailleurs la recherche connexe à ces cours (thèses de M.A., etc.), même si elle est parfois descriptive, atteint rarement l'explication. Vous savez bien que le thème le plus souvent choisi par les étudiants en maîtrise chez nous, est de dresser le simple glossaire bilingue d'un domaine du discours hautement spécialisé. Ainsi on recueille beaucoup de données terminologiques, mais avec une conception théorique restée primitive. Par conséquent, même un ouvrage canadien aussi réputé que la Stylistique comparée de vinay et Darbelnet ${ }^{9}$ (cité par Ljudskanov) reste borné à cause des objectifs de 1 'enseignement professionnel.

Il faut que nos départements de linguistique acceptent, tôt ou tard, que la traductologie fasse partie de leur discipline et de leurs enseignements; et, en outre, que l'objet primordial de cette traductologie soit la traduction nature1le, traitée d'une manière descriptive et explicative.

Deux éminents Itnguistes, 1 'un russe, l'autre américain, oeuvrant dans le cadre de travaux en traduction automatique, affirment l'importance de la traductologie pour la linguistique. Volci d'abord une citation de Melchuk.

What then should be the character of subsequent work on AT [Automatic Translation] ? There is only one possible answer : the elaboration of operational models of language, logical systems giving multiple-meaning equivalence between text and meaning in both directions. The study and description of the relationship text-to-

9. J.-P. Vinay et J. Darbelnet, Sty Zistique comparée du français et de l'anglais, Montréal, Beauchemin, 1962. 
meaning in all its aspects (including the historical, social, psysiological and other aspects) has always been recognized as the central problem of the science of language. The problem of AT thus coincides with the problem of synchronic linguistics as such. This is confirmed in particular by the fact that most 1inguistic papers published an AT from 1963 to 66 are essentially ordinary linguistic work [..] Contrary to the mistaken view that iAT takes account only of the external characteristics of text and completely disregards the meaning, sooner or later researchers find that meaning occupies the forefront of their attention; moreover, any translation is before all else the transmission of meaning by a transformation retaining the sensel0.

Pour Melchuk, la traduction automatique est un apport à la linguistique théorique parce qu'elle conduit à la construction des modèles formels de la traduction; la traductologie est importante parce qu'elle oblige à une étude du rapport entre le texte et la signification de ce texte (ou vice-versa, lorsqu'on conçoit le texte-cible). Le rapport entre le texte et la signification du texte correspond peut-être à une définition globale, linguistique, de toute la sémantique.

On peut tirer la conclusion suivante des propos de Melchuk. Si, en linguistique, d'aucuns se sont permis d'étudier la phonologie comme un code en soi, de séparer la syntaxe de 1a sémantique, d'établir le lexique indépendamment de la syntaxe, en traductologie, au contraire, il faut nécessairement abolir ce cloisonnement : tous les niveaux énumérés par Ljudskanov ${ }^{11}$, graphique, phonétique, sémique, morphologique, syntaxique et sémantique, s'intègrent. Voici la deuxième citation à ce propos :

Although theoretical research is concerned with the totality of linguistic competence, actual instances of such research activities usually focus on particular aspects of this totality. The general approach is that of deduction. Thus, for example, a proposed explanation for complementation in a language in general does not

10. I.A. Melchuk, 1967, "Linguistics and Automatic Translation", dans Intemational Social Science Joumal, vol. $19, \mathrm{n}^{\circ} 1$.

11. A. Ljudskanov, 1969, Traduction humaine et traduction automatique, Paris, Dunot, fascicule 2, p. 3. 
exhaustively take into consideration all of the verbs in the language. Furthermore, in practice, the rules proposed are never exhaustively crosschecked against others in the language. On the other hand research into experimental MT and other activities in computational linguistic must be constantly concerned with the total range of exhaustive application of the results of the more theory-oriented research. Thus inadequacies in the theory oriented descriptions are frequently and constantly unearthed by the more exhaustive concerns of computational linguistics and MT. This underlines the fact that research in MT, not only in theory but also in practice, is concerned with the totality of the descriptive adequacy of the grammar ${ }^{12}$.

En somme, si nous prenons ces deux citations ensembles, il parait que la traductologie et la traduction automatique nous force à compléter les modèles linguistiques dans deux dimentions. D'abord, en profondeur, en intensité (voir Melchuk) et, ensuite, en extention, en "exhaustiveness" (voir la dernière citation).

À ces deux opinions, nous nous permettons d'ajouter la nôtre : 1a traductologie représente le terrain le plus riche pour la linguistique comparative sur le plan de la synchronie.

Pour comprendre pourquoi on traduit, pourquoi on fait des changements lors d'une traduction, il faut tenir compte des différences entre les langues. En apercevant ces différences, on devient conscient des particularités, de chacune des langues impliquées, ainsi que des similitudes. On ne peut faire de la linguistique générale avec la connaissance $d^{\prime}$ une seule langue. On resterait prisonnier, psychologiquement, des structures de cette langue. La richesse de la linguistique structurale américaine était due, en grande mesure, à l'importance que cette école accordait à l'étude des langues amérindiennes. Or, de cette différenciation, le linguiste falt continuellement, inconsciemment parfois, des comparaisons par vole de traduction naturelle. À fortiori, une traductologie symématique et formalisée permettrait de miner beaucoup plus de cette velne. Mentionnons, à titre d'exemple, les problèmes soulevés

12. W. S.-Y. Wang, B.K. T'sou, S.W.C. Chen et al., 1971, Research in Chinese-English Machine Translation, U.S. Air Force Rome Air Devel opment Center, p. 7-8. 
lors de la traduction en français des groupes nominaux qui ne comportent ni article ni déictique dans la langue source.

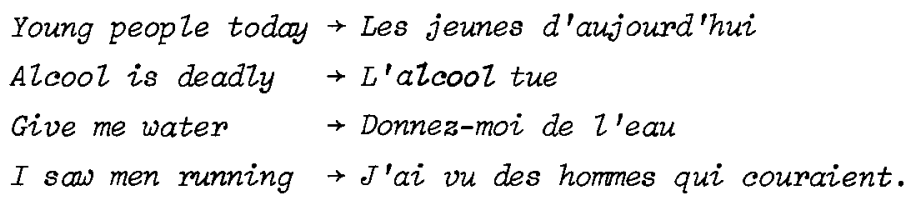

Du moins dans ces exemples il n'est pas question de stylistique mais de la grammaire la plus élémentaire et obligatoire. Donc c'est un niveau qui n'intéresse guère le traducteur professionnel, qui a dû le maîtriser avant même de songer à devenir traducteur. Dans l'enselgnement du français comme langue seconde, on l'enseigne par méthode inductive. (Autrefois, on employait pour l'enseignement des exemples de traduction pareils à ceux que nous venons de donner.) Dans les deux cas, l'explication manque, et c'est pourquoi il faut aborder une description explicite au moment où $1^{\prime}$ on veut faire la traduction automatique anglais-français. Alors, on arrive rapidement à la conclusion que le français exige presque toujours un déictique, soit défini, soit indéfini et, en outre, que 1 'article défini possède deux fonctions, 1'une définie (proprement dite),

\section{L'homme que je vois devant moi,}

et 1'autre générique :

\section{Dieu créa l'homme / les hommes.}

Ce qui nous ramène a l'analyse de 1'anglais, car il faut, pour les fins de traduction, décortiquer le mécanisme par lequel 1 'opposition "définiegénérique"s'exprime dans la langue source. Or, comme Chafe le démontrait dans un des tout premiers ouvrages sur la "sêmantique générative", 1 'analyse de cette opposition en anglais s'avère très complexe ${ }^{13}$.

13. W.L. Chafe, English Noun Inflection and Related Matters from a Generative Semantic Point of View, PEGS Paper $n^{\circ} 15,1967$. Notre collègue au groupe TAUM, Jules Dansereau, a trouvé des règles de transfert superficielles qui permettent une traduction correcte dans la majorité des cas, mais qui ne sont pas encore "exhaustives". 
Jakobson a soutenu l'hypothèse, d'ailleurs reprise par Ljudskanov, selon laquelle ce qui est traduit, c'est 1a signification; et réciproquement, toute représentation de la signification est nécessairement une traduction ${ }^{14}$.

D'autres modèles ont prévalu quant à l'importance qu'ils accordaient à l'intégration de la sémantique. Ce fut le cas de la grammaire stratificationnelle de Lamb élaborée alors qu'il travaillait à Berkeley avec un groupe de recherches sur la traduction automatique ${ }^{15}$. On connaît encore, à Stanford, le groupe de Schank ${ }^{16}$, qui travaille depuis quatre ans sur 1'intelligence artificielle et s'est tourné depuis vers la traduction automatique. Plus récemment, Y. Wilks, dans une communication intitulee An Artificial Intelligence Approach to Tronslation, parle du type de sémantique qu'il faut incorporer dans de telles recherches et surtout la nécessité, selon Winograd ${ }^{17}$, d'incorporer un composant capable de tirer des déductions ${ }^{18}$. Il ne faut pas mésestimer, en outre, les travaux du groupe dirigé par Yngve qui, durant les années soixante, fut le premier à s'attaquer à la traduction automatique du langage naturel dans la notation du calcul des prédicats ${ }^{19}$.

14. R. Jakobson, 1959, "On Iinguistic aspects of translation" dans Brower, On Translation, Cambridge, Harvard University Press.

15. S. M. Lamb, 1966, Outiine of Stratificational Grammar, Georgetown University Press.

16. R. C. Schank, 1969, A Conceptual Dependency Representation for Computer Oriented Semantics, Stanford, Artificial Intelligence Project.

17. T. Winograd, 1971, Procedures as a Representation for Data in a Computer Program for Understanding Natural Language, Project MAC, M.I.T.

18. Y. Wilks, 1961, An Artificial IntelZigence Approach to Machine Translation, Stanford University, Computer Science Department.

19. V. H. Yngve, 1965, M. T. at M. I. T., M.I.T. 
En Europe, la grammaire corrélationnelle de Ceccato fut conçue pour la traduction automatique 20 . La théorie de Shillan, du groupe de Cambridge, permettait d'établir une corrélation directe entre 1a sémantique et certains paramètres de la phonétique, c'est-à-dire certains traits prosodiques comme 1 'accent et 1 'intonation en anglais 21 . On peut voir, à travers cet aperçu de la dernière décennie, comment la traduction automatique a revivifié la traductologie dans le cadre de l'informatique, qui encourageait les chercheurs à élaborer des modèles formels qui ouvriraient une voie à la sémantique.

Dans une conférence récente à $I^{\prime}$ Université McGil122, Sydney Lamb parla du réseau conceptuel sur lequel se brancherait la grammaire d'une langue. D'après Lamb, ces réseaux sont pourvus en partie de concepts et de mécanismes opérationnels extra-linguistiques, parfois universaux; mais, en majorité, les concepts s'apprennent, entrent dans le réseau, par 1'intermédiaire de la langue. Par conséquent, le réseau conceptuel de 1a personne qui aurait appris ces concepts par une 1angue donnée $L_{1}$ diffère en quelque sorte du sujet qui 1 'aurait appris par une autre langue $L_{2}$.

On revient à 1 'hypothèse de Whorf. Les traducteurs et les traductologues en connaissent déjà une preuve. Il arrive assez souvent qu'une traduction qui est correcte selon la grammaire et selon le dictionnaire boite à cause des différences du raccordement du concept dans le réseau total engendré par $L_{1}$ ou $L_{2}$.

Prenons un exemple très proche, le mot national dans un usage tel que l'assemblée nationale qui siège au parlement de québec. Bien que les dictionnaires du français et de 1'anglais, tout comme la syntaxe qui se rattache au mot, semblent indiquer le mot anglais national comme tra-

20. S. Ceccato, 1967, "Correlational Analysis", dans A.D. Booth, Machine Translation, North Holland Company, Amsterdam.

21. D. Shillan, 1967, "Detecting meaning through speech", META, II, 3 : 85-88.

22. Language, Thought and Concept, 15 avri1 1972. 
duction de national, c'est un faux aml car $1^{\prime}$ emplacement du concept sous-jacent à national dans le réseau conceptuel de l'anglophone est légèrement déplacé par rapport à la situation de national dans le réseau du franco-canadien. Il serait plus fidèle, à notre avis (avis d'un anglais, bien sûr) de traduire assemblée nationale par popular assembly. Il est trop tard pour le faire, évidement.

Pourtant, 1'argument le plus pressant pour l'inclusion d'une composante sémantique dans tout modèle de traduction nous semble celui qu'expose Wilks dans son dernier livre 23 , comme dans plusieurs de ses écrits antérieurs qui remontent jusqu'à 1 'époque de son association avec le groupe de Masterman à Cambridge. En bref, cet argument repose sur la nécessité de distinguer entre différents sens d'un même mot. Un exemple typique serait le mot "post" dans la phrase anglaise :

She went to the post.

Est-ce que nous devons traduire ce mot par la "poste", le "poteau", ou même, s'il s'agit d'une jument de course, la "ligne de départ". (Ici nous supposons que dans le cadre de la traduction, on peut faire une équivalence entre "différence de sens" et "différence de production".) Dans une proportion limitée de cas, ces ambiguités, on homographies, peuvent être résolues à l'intérieur de la phrase par des moyens mi-syntaxiques, mi-sémantiques, tels que ceux que nous avons appliqués dans le système TAUM. Pour nous, le critère de décision est la collocation d'un verbe avec une certaine catégorie de sujet ou de complément. Par exemple, know se traduit savoir avec un complément de phrase enchâssée, mais connaitre avec d'autres catégories d'objet ${ }^{24}$ (il en est ainsi grosso modo, bien sûr). Mais la considération irréfutable présentée par Wilks, est que très souvent le sens d'un mot ne se décide par aucun élément de la phrase qui le contient : il faut procéder à une sémantique

23. Y. Wilks, 1971, Grommar, Meaning and the Machine Analysis of Language, Londres, Routledge.

24. B. Harris, 1969, "Lexicography with a W-Grammar", Recherche sur Za traduction automatique, no 12 . 
146 problèmes de sémontique

du texte entier, en appliquant des critères de consistance générale entre toutes les propositions du texte ${ }^{25}$. Ainsi 11 est amené à critiquer non seulement les limites imposêes par les grammalriens transformationnels en faveur de la syntaxe et au détriment de la sémantique, mais aussi 1'effort de poser toute la gramaire sur la grammire de la phrase isolée. Ce sont des expériences analogues qui nous ont amené au groupe TAUM, à faire quelques essais de traduction en utilisant les réseaux sémantiques et cumulatifs proposés par Hofmann ${ }^{26}$ (Réseaux-C).

Brian Harris

Université de Montréal

25. Voir aussi I. Bellert, 1972, Sets of Implications as the Interpretive Component of a Grammar, Montréal, Université de Montréal, Groupe de linguistique formelle.

26. Voir p. 19-38, article de T. R. Hofmann. 